\title{
Transmission spectroscopy of the sodium doublet in WASP-17b with the VLT
}

\author{
Patricia L. Wood ${ }^{1}$ and Pierre F. L. Maxted ${ }^{1}$ \\ ${ }^{1}$ Keele University, Staffordshire ST5 5BG, UK \\ email: plw@astro.keele.ac.uk
}

\begin{abstract}
The detection of sodium absorption during primary transit implies the presence of an atmosphere around an extrasolar planet. WASP-17b (Anderson et al. 2010a) is the least dense known planet, with a radius twice that of Jupiter. It orbits an F6-type star, and its low gravity gives its atmosphere a very large scale height. The sodium transit depth is expected to be $4.1-$ 5.2 times deeper than for HD 209458b (Seager \& Sasselov 2000). We obtained 24 spectra with the GIRAFFE spectrograph on the VLT, 8 during transit. We measured the flux in the sodium doublet at $5889.95 \AA$ and $5895.92 \AA$ using bandpasses $0.75,1.5,3.0$ and $6.0 \AA$. We find a transit depth of $0.55 \pm 0.13 \%$ at $1.5 \AA(4.3 \sigma)$. WASP-17b therefore has an atmosphere which is depleted in sodium compared to predictions.
\end{abstract}

Keywords. planetary systems, stars: individual (WASP-17), techniques: spectroscopic

\section{Transmission Spectroscopy}

When light from a star passes through the atmosphere of an orbiting planet during transit, some wavelengths are absorbed much more strongly than others. The planet will appear larger at these wavelengths, i.e., the transit will be slightly deeper at wavelengths where there is an opacity source in the upper atmosphere. Measurements at wavelengths where the opacity is high, e.g. narrow bandwidths around the NaI lines, probe the upper layers of the atmosphere, whilst wider bandwidths probe the lower atmosphere. $\mathrm{Na}$ absorption has so far only been detected in the atmospheres of the two exoplanets HD 189733b (Redfield et al. 2008) and HD 209458b (Snellen et al. 2008). As the atmosphere of WASP-17b has a large scale height, the sodium transit depth is predicted to be $4.1-5.2$ times as large as that for HD $209458 \mathrm{~b} ; 0.135 \%$ at bandwidth $1.5 \AA$.

\section{Observations}

- 24 spectra obtained with VLT GIRAFFE spectrograph

- IFU feed on WASP-17 and comparison star

- 8 spectra in-transit

- $5821-6146 \AA$, $0.48 \AA$ resolution, $0.08 \AA$ per pixel

- Custom-made optimal extraction software

- Telluric lines removed using synthetic telluric absorption spectra

Table 1. Parameters of WASP-17b and HD 209458b for comparison

\begin{tabular}{lccccrc}
\hline Planet & Period, d & Mass, $\mathbf{M}_{J}$ & Radius, $\mathbf{R}_{J}$ & $\mathbf{T}_{e q}, \mathbf{K}$ & $\mathbf{H}, \mathbf{k m}$ & Spec. Type \\
\hline WASP-17b $^{1}$ & 3.74 & 0.49 & 2.00 & 1773 & 2300 & F6V \\
HD 209458b & 3.52 & 0.64 & 1.35 & 1300 & 500 & G0V \\
\hline
\end{tabular}

${ }^{1}$ Parameters taken from Anderson et al. 2010b, in prep. 


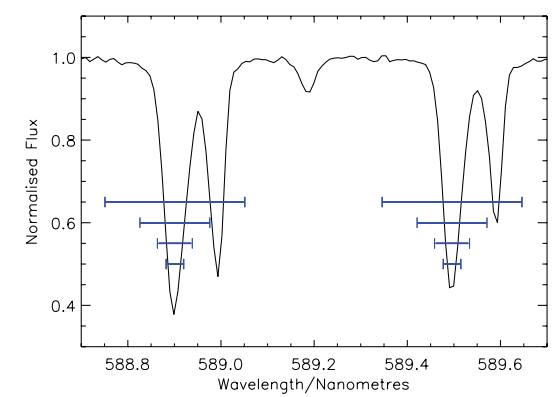

Figure 1. Part of a normalised spectrum of WASP-17 taken with GIRAFFE, showing the $\mathrm{Na}$ lines at $5890 \AA$ and $5895 \AA$. The shallower lines in each pair are interstellar Na absorption features. Measured bandwidths are marked in blue: $0.75 \AA, 1.5 \AA, 3.0 \AA, 6.0 \AA$.

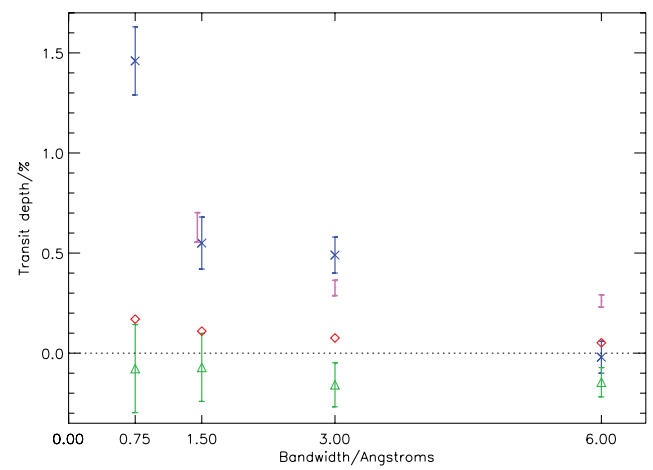

Figure 2. Na transit depths for WASP-17 (blue). In pink are values for HD 2094585 , scaled up by the difference in scale height. Identical measurements on a comparison star observed simultaneously with the same instrument (green triangles) show that the level of systematic errors on these measurements is low. Red diamonds are photon noise.

Table 2. Sodium transit depths for WASP-17b measured at 4 different bandwidths

\begin{tabular}{l|cccc}
\hline Bandwidth $(\AA)$ & $\mathbf{0 . 7 5}$ & $\mathbf{1 . 5}$ & $\mathbf{3 . 0}$ & $\mathbf{6 . 0}$ \\
Transit Depth $(\%)$ & $1.46 \pm 0.017$ & $0.55 \pm 0.13$ & $0.49 \pm 0.09$ & $-0.019 \pm 0.076$ \\
\hline
\end{tabular}

\section{Results and Conclusions}

We find that, like HD 209458b, WASP-17b has an atmosphere depleted in sodium compared to predictions, particularly in the $6 \AA$ bandpass that probes the lower depths of the atmosphere. This may be due to photo-ionization, condensation, or the presence of high altitude clouds or haze in the atmosphere. We will now be investigating which is the more likely explanation.

\section{References}

Anderson, D. R., et al. 2010a, ApJ, 709, 159

Anderson, D. R., et al. 2010b, in prep.

Seager, S. \& Sasselov, D. D. 2000, ApJ, 540, 504

Redfield, S., Endl, M., Cochran, W. D., \& Koesterke, L. 2008, AJ, 673, L87

Snellen, I. A. G., Albrecht, S., de Mooji, E. J. W., \& Le Poole, R. S. 2008, A\&̈A, 487, 357 